\title{
STABILITY OF THE CONFIGURATIONS OF POINT VORTICES ON A SPHERE
}

\author{
V. V. Meleshko, ${ }^{1}$ P. K. Newton, ${ }^{2}$ and V. V. Ostrovs'kyi ${ }^{1}$ \\ UDC 539.3
}

We study the motion of point vortices on a sphere and, using the methods of linear algebra, find the symmetric configurations of relative equilibrium. Furthermore, we give a catalog of symmetric configurations based on regular polyhedrons. Finally, we investigate the stability of the equilibrium configurations found.

\section{Introduction}

Vortex dynamics as a broad branch of present-day fluid dynamics springs from the classical memoir by Helmholtz [21]. In this work, Helmholtz not only defined vortex motion from the kinematic viewpoint (before him, Stokes did this in 1845), but also brought to perfection the dynamic theory of vortex motion in an ideal (inviscid) incompressible fluid in the form of three conservation laws. These laws were written approximately in the same way as they are given in all textbooks on fluid dynamics. The main sense of dynamic theory lies in the law of conservation of vortex motion: in an ideal fluid, vortices cannot disappear, and their intensity is constant in time. The conservation of vortices in time is supplemented by their conservation in space: vortex intensity is constant along every vortex tube, and these tubes either are closed or reach the boundary of fluid. Up to now, a vast amount of literature on these problems has been accumulated (a fairly complete review of early publications is presented in [28]), including both classical textbooks on fluid mechanics and special monographs on vortex dynamics $[1,3,6,10,12,15,16,29]$.

Among different problems in this field, the dynamics of point vortices on a plane, i.e., the interaction of parallel vortex filaments, is especially interesting. At first glance, the Hamilton equations of motion of such point vortices, derived first in the book [9], are purely kinematic conditions of the motion of a point, where the vortex is situated, in the velocity field induced by other vortices. However, as noted in [8, Sec. 21], these equations express the dynamic laws of motion, which follow from the Euler equations of motion for an ideal incompressible fluid.

Until recently, significantly less attention was devoted to the motion of point vortices on a sphere. Gromeka [7] was the first who began to study such a motion of vortices. Using cartographic projection, he constructed the general equations of vortex motion on a cylinder and a sphere. Gromeka also considered a more general problem of vortex motion in a closed fixed domain on a sphere. However, his equations contain the unknown "Green function for the volume occupied by the fluid." Unfortunately, this work remained almost unnoticed [5].

Unlike Gromeka, Zermelo [34] derived the complete equations of motion. Although these equations were obtained at the beginning of the 20th century, the problem of dynamics of vortex structures on a sphere did not attract sufficient attention at that time. The same equations, after 70 years of disregard, were repeatedly derived by Bogomolov in [2]. He derived the basic equations of motion, showed that the system is Hamiltonian, and found the invariants of motion. Bogomolov also deduced the equations of motion of point vortices on a moving

\footnotetext{
${ }^{1}$ Kyiv National University, Kyiv, Ukraine.

${ }^{2}$ University of South California, Los Angeles, USA.
}

Translated from Matematychni Metody ta Fizyko-Mekhanichni Polya, Vol.52, No.3, pp.146-159, July-September, 2009. Original article submitted February 6, 2009. 
sphere. A fairly brief derivation of the equations of motion of point vortices is presented in [24]. Here, the equations of motion of homogeneous vorticity domains were also obtained. The method of deriving the equations of motion on a surface with constant curvature was generalized in [23].

The problems of stability of vortex configurations on both a sphere and a plane have been studied insufficiently. Numerous works of this direction are devoted to studying the stability of vortices placed at the vertices of regular polygons [25,27]. In these works, the stability of vortex rings with identical or opposite, as to their sign, vortex intensities is investigated. A more complex problem of the stability of a vortex ring with a vortex on the pole is studied in [20]. The method of energy and moment is used in [32] for the investigation of nonlinear stability of three vortices with different intensities on a sphere. The paper [11] is the only work devoted to studying configurations formed by regular polyhedrons.

In the present work, we concentrate our attention on finding the symmetric configurations of relative equilibrium of point vortex structures on a sphere and studying the stability of degenerate equilibrium configurations.

\section{Motion of Point Vortices on a Sphere}

A vortex field $\omega$ is defined as the rotor of a velocity field $\mathbf{u}$ :

$$
\omega=\nabla \times \mathbf{u} .
$$

In the case where a velocity field describes the motion of an incompressible homogeneous ideal fluid, the principle of mass conservation yields

$$
\nabla \cdot \mathbf{u}=0
$$

It is easy to verify that, in the case where the fluid under consideration moves along the surface of a sphere of unit radius, we may introduce a stream function $\psi$, which defines the velocity field in the following way:

$$
\mathbf{u}=(\nabla \psi) \times \mathbf{e}_{r},
$$

where $\mathbf{e}_{r}$ is a unit normal vector directed along the sphere radius. Indeed,

$$
\nabla \cdot \mathbf{u}=\nabla \cdot\left((\nabla \psi) \times \mathbf{e}_{r}\right)=\mathbf{e}_{r} \cdot(\nabla \times(\nabla \psi))-\nabla \psi \cdot\left(\nabla \times \mathbf{e}_{r}\right)=\mathbf{e}_{r} \cdot \mathbf{0}-\nabla \psi \cdot \mathbf{0}=0,
$$

i.e., condition (2) is satisfied automatically. The level lines of function $\psi$ will be streamlines as well, i.e., the lines along which fluid particles will move in the velocity field $\mathbf{u}$.

Substituting (3) in (1), we obtain

$$
\Delta \psi=-\omega,
$$

where $\boldsymbol{\omega}=\boldsymbol{\omega} \mathbf{e}_{r}$. In spherical coordinates, this relation will have the form

$$
\frac{1}{\sin \theta} \frac{\partial}{\partial \theta}\left(\sin \theta \frac{\partial \psi}{\partial \theta}\right)+\frac{1}{\sin ^{2} \theta} \frac{\partial^{2} \psi}{\partial \varphi^{2}}=-\omega
$$


Rewriting relation (3) in spherical coordinates, we come to the following form of velocity field:

$$
\mathbf{u}=\left(0, \frac{1}{\sin \theta} \frac{\partial \psi}{\partial \varphi},-\frac{\partial \psi}{\partial \theta}\right)
$$

The operator on the left-hand side of equation (6) is called a Beltrami-Laplace operator. For the Kelvin theorem of circulation to be true, i.e., in order that

$$
\int_{S} \omega d S=0
$$

where $S$ is the sphere surface, we define the Green function $G\left(\theta, \varphi, \theta^{\prime}, \varphi^{\prime}\right)$ for the Beltrami-Laplace operator as

$$
\frac{1}{\sin \theta} \frac{\partial}{\partial \varphi}\left(\sin \theta \frac{\partial G}{\partial \theta}\right)+\frac{1}{\sin ^{2} \theta} \frac{\partial^{2} G}{\partial \varphi^{2}}=\delta\left(\theta, \varphi, \theta^{\prime}, \varphi^{\prime}\right)-\frac{1}{4 \pi}
$$

The last term on the right-hand side corresponds to a constant vorticity field, whose integral circulation is equal to -1 , i.e., is equal and opposite in sign to the intensity of singularity at the point $\left(\theta^{\prime}, \varphi^{\prime}\right)$. This singularity is called a point vortex on a sphere.

It is easy to verify that the function

$$
G\left(\theta, \varphi, \theta^{\prime}, \varphi^{\prime}\right)=-\frac{1}{2 \pi} \ln \sin \left(\frac{1}{2} \gamma\left(\theta, \varphi, \theta^{\prime}, \varphi^{\prime}\right)\right)
$$

where $\gamma\left(\theta, \varphi, \theta^{\prime}, \varphi^{\prime}\right)$ is the central angle between points with coordinates $(\theta, \varphi)$ and $\left(\theta^{\prime}, \varphi^{\prime}\right)$, satisfies equation (9). Using the trigonometric relation for the sine of half-angle, we obtain

$$
G=-\frac{1}{4 \pi} \ln (1-\cos \gamma) .
$$

This relation is more convenient since $\cos \gamma$ can be expressed as

$$
\cos \gamma=\cos \theta \cos \theta^{\prime}+\sin \theta \sin \theta^{\prime} \cos \left(\varphi-\varphi^{\prime}\right) .
$$

Using relation (11) for the Green function, we can find the stream function $\psi$ as the solution of Eq. (6):

$$
\psi(\theta, \varphi)=\int_{S} \omega\left(\theta^{\prime}, \varphi^{\prime}\right) \ln (1-\cos \gamma) \sin \theta^{\prime} d \theta^{\prime} d \varphi^{\prime}
$$

For obtaining the equations of motion of $N$ point vortices on a fixed sphere, we choose $\omega$ in the following way: 


$$
\omega(\theta, \varphi)=\sum_{i=1}^{N} \Gamma_{i} \delta\left(\theta, \varphi, \theta_{i}, \varphi_{i}\right),
$$

where $\Gamma_{i}$ is the intensity of the $i$ th vortex. Substituting (14) in (13) and using (7), we obtain

$$
\begin{gathered}
u_{\theta}=\frac{1}{\sin \theta} \frac{\partial \psi}{\partial \varphi}=-\frac{1}{4 \pi} \sum_{j=1}^{N} \Gamma_{j} \frac{\sin \theta_{j} \sin \left(\varphi-\varphi_{j}\right)}{1-\cos \gamma_{j}}, \\
u_{\varphi}=-\frac{\partial \psi}{\partial \theta}=-\frac{1}{4 \pi} \sum_{j=1}^{N} \Gamma_{j} \frac{\cos \theta \sin \theta_{j} \cos \left(\varphi-\varphi_{j}\right)-\sin \theta \cos \theta_{j}}{1-\cos \gamma_{j}},
\end{gathered}
$$

where $\gamma_{j}=\gamma\left(\theta, \varphi, \theta_{j}, \varphi_{j}\right)$.

According to the Helmholtz theorem, the vortices under study will move as fluid particles, i.e., the equations of motion of $N$ point vortices have the form

$$
\begin{gathered}
\dot{\theta}_{i}=-\frac{1}{4 \pi} \sum_{j=1, i \neq j}^{N} \Gamma_{j} \frac{\sin \theta_{j} \sin \left(\varphi_{i}-\varphi_{j}\right)}{1-\cos \gamma_{i j}}, \\
\sin \theta_{i} \varphi_{i}=-\frac{1}{4 \pi} \sum_{j=1, i \neq j}^{N} \Gamma_{j} \frac{\cos \theta_{i} \sin \theta_{j} \cos \left(\varphi_{i}-\varphi_{j}\right)-\sin \theta_{i} \cos \theta_{j}}{1-\cos \gamma_{i j}},
\end{gathered}
$$

where $\gamma_{i j}=\gamma\left(\theta_{i}, \varphi_{i}, \theta_{j}, \varphi_{j}\right)$.

The equations given above can be written in vector form [29]:

$$
\dot{\mathbf{x}}_{i}=\sum_{j=1, j \neq i}^{N} \frac{\Gamma_{j}}{2 \pi} \frac{\mathbf{x}_{j} \times \mathbf{x}_{i}}{\left(\mathbf{x}_{i}-\mathbf{x}_{j}\right)^{2}} .
$$

Here, $\mathbf{x}_{i}$ is the vector connecting the sphere center with the point where a point vortex is located, i.e., $\left\|\mathbf{x}_{i}\right\|=1$.

For obtaining the equations of motion of point vortices on a rotating sphere, it is necessary to add $\omega_{r}=2 \Omega \cos \theta$ to the field $\omega$ determined in (14). Then the equations of motion in vector form will look like

$$
\dot{\mathbf{x}}_{i}=\sum_{j=1, j \neq i}^{N} \frac{\Gamma_{j}}{2 \pi} \frac{\mathbf{x}_{j} \times \mathbf{x}_{i}}{\left(\mathbf{x}_{i}-\mathbf{x}_{j}\right)^{2}}+\Omega \mathbf{e}_{z} \times \mathbf{x}_{i}
$$

where $\mathbf{e}_{z}=(0,0,1)$.

Note that Eqs. (16) can be represented in the form of Hamilton equations with the following Hamiltonian and canonical variables: 


$$
\begin{gathered}
H=\frac{1}{4 \pi} \sum_{i<j} \Gamma_{i} \Gamma_{j} \ln \ell_{i j}, \\
P=\sqrt{\left|\Gamma_{i}\right|} \cos \theta_{i}, \quad Q=\sqrt{\left|\Gamma_{i}\right|} \varphi_{i}, \\
\dot{P}_{i}=\frac{\partial H}{\partial Q_{i}}, \quad \dot{Q}_{i}=-\frac{\partial H}{\partial P_{i}},
\end{gathered}
$$

where $\ell_{i j}^{2}=\left(\mathbf{x}_{i}-\mathbf{x}_{j}\right)^{2}=2\left(1-\cos \gamma_{i j}\right)$.

The Poisson bracket for this Hamiltonian system has the form

$$
\{f, g\}=\sum_{k=1}^{N} \frac{1}{\Gamma_{k}}\left(\frac{\partial f}{\partial \cos \theta_{k}} \frac{\partial g}{\partial \varphi_{k}}-\frac{\partial f}{\partial \varphi_{k}} \frac{\partial g}{\partial \cos \theta_{k}}\right), \quad\left\{\varphi_{i}, \cos \theta_{j}\right\}=\frac{\delta_{i j}}{\Gamma_{i}}
$$

where $\delta_{i j}$ is the Kronecker symbol.

Along with the Hamiltonian $H$, this system admits three more integrals of motion:

$$
\begin{gathered}
M_{1}=\sum_{i=1}^{N} \Gamma_{i} \sin \theta_{i} \cos \varphi_{i}=\text { const }, \\
M_{2}=\sum_{i=1}^{N} \Gamma_{i} \sin \theta_{i} \sin \varphi_{i}=\text { const, } \\
M_{3}=\sum_{i=1}^{N} \Gamma_{i} \cos \theta_{i}=\text { const }
\end{gathered}
$$

which together form a vector

$$
\mathbf{M}=\left(M_{1}, M_{2}, M_{3}\right)=\sum_{k=1}^{N} \Gamma_{k} \mathbf{x}_{k}
$$

called the vorticity moment [29]. It is also useful to introduce a vector called the vorticity center:

$$
\mathbf{c}=\frac{\mathbf{M}}{\Gamma}, \quad \text { where } \quad \Gamma=\sum_{i=1}^{N} \Gamma_{i}
$$

Note that $H, M_{1}, M_{2}$, and $M_{3}$ in the general case give only three independent involutive integrals:

$$
\left\{H, M_{3}\right\}=0, \quad\left\{H, M_{1}^{2}+M_{2}^{2}\right\}=0, \quad\left\{M_{3}, M_{1}^{2}+M_{2}^{2}\right\}=0 .
$$


In the case where $\mathbf{M}=\mathbf{0}$, using relations

$$
\left\{M_{2}, M_{1}\right\}=M_{3}, \quad\left\{M_{1}, M_{3}\right\}=M_{2}, \quad\left\{M_{3}, M_{2}\right\}=M_{1},
$$

we obtain four independent involutive integrals. We formulate these facts in the form of a theorem, which was proved in [29].

Theorem 1. The problem of motion of three vortices on a sphere is completely integrable for vortices of an arbitrary intensity. If the vorticity center is equal to zero, the problem of motion of four vortices on a sphere is also integrable.

In 1998, an analogous result was obtained independently by Borisov and Lebedev in [3, 18, 19]. However, as was noted by Borisov, this result was first established in Zermelo's thesis, which was published at the beginning of the 20th century [4, 34].

An alternative derivation of the equations of motion can be found in [2, 3, 13, 34]. The equations of motion in stereographic projection and the limiting process to a plane case are presented in $[14,29]$.

\section{Equilibrium Configurations}

For finding equilibrium configurations, we use an approach that will enable us to determine not only the configurations of absolute equilibrium, but also the configurations of relative equilibrium. To simplify our considerations, we use the equations of motion in vector form (18). Consider a system of $N$ vortices of an arbitrary intensity. The motion of the $i$ th and $j$ th vortices is described by

$$
\begin{gathered}
\dot{\mathbf{x}}_{i}=\sum_{k=1, k \neq i}^{N} \frac{\Gamma_{k}}{2 \pi} \frac{\mathbf{x}_{k} \times \mathbf{x}_{i}}{\left(\mathbf{x}_{i}-\mathbf{x}_{k}\right)^{2}}+\Omega \mathbf{e}_{z} \times \mathbf{x}_{i}, \\
\dot{\mathbf{x}}_{j}=\sum_{k=1, k \neq j}^{N} \frac{\Gamma_{k}}{2 \pi} \frac{\mathbf{x}_{k} \times \mathbf{x}_{j}}{\left(\mathbf{x}_{j}-\mathbf{x}_{k}\right)^{2}}+\Omega \mathbf{e}_{z} \times \mathbf{x}_{j} .
\end{gathered}
$$

Subtracting the second equation from the first and multiplying by $\left(\mathbf{x}_{i}-\mathbf{x}_{j}\right)$, we obtain after algebraic transformations an equation for $\ell_{i j}^{2}=\left(\mathbf{x}_{i}-\mathbf{x}_{j}\right)^{2}$ :

$$
\frac{d \ell_{i j}^{2}}{d t}=\sum_{k=1, k \neq i, k \neq j}^{N} \Gamma_{k} \frac{V_{i j k}}{\pi}\left(\frac{1}{\ell_{i k}^{2}}-\frac{1}{\ell_{j k}^{2}}\right),
$$

where $V_{i j k}=\mathbf{x}_{i} \cdot \mathbf{x}_{j} \times \mathbf{x}_{k}$.

We find the configurations of absolute and relative equilibrium in the problem of motion of $N$ point vortices as the equilibrium position of system (26), i.e., taking

$$
\frac{d \ell_{i j}^{2}}{d t}=0
$$



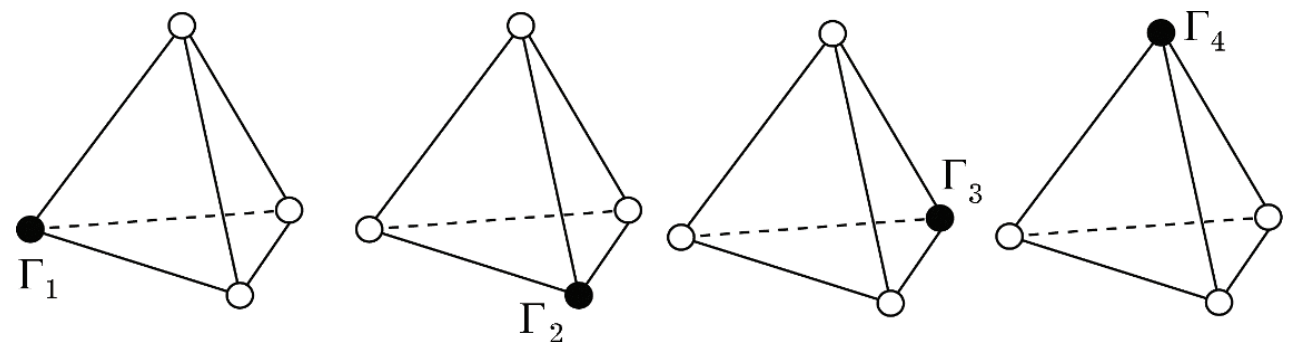

Fig. 1. Set of independent intensities generating an equilibrium configuration in the form of a tetrahedron.

and then obtain from (26)

$$
\sum_{k=1, k \neq i, k \neq j}^{N} \Gamma_{k} \frac{V_{i j k}}{\pi}\left(\frac{1}{\ell_{i k}^{2}}-\frac{1}{\ell_{j k}^{2}}\right)=0 .
$$

Further, we rewrite these equations in convenient matrix form:

$$
\mathbf{A} \Gamma=0,
$$

where $\mathbf{A}$ is a matrix of size $\frac{N(N-1)}{2} \times N$ with elements

$$
A_{l k}=\frac{V_{i j k}}{\pi}\left(\frac{1}{\ell_{i k}^{2}}-\frac{1}{\ell_{j k}^{2}}\right), \quad l=i+j, \quad i, j, k=1, \ldots, N
$$

and $\Gamma=\left(\Gamma_{1}, \ldots, \Gamma_{N}\right)$ is the vector of vortex intensities.

If the intensities $\Gamma$ are known, the problem of finding equilibrium configurations in such a formulation is quite complex: indeed, for its solution, it is necessary to determine all matrices $\mathbf{A}$ such that their kernels contain the vector $\Gamma$ assigned beforehand. Afterwards, it is necessary to establish which matrices of the found ones contain the structure assigned by relation (28). Finally, one should solve the nonlinear system of equations for $\ell_{i j}$.

However, looking at this problem from the other side, i.e., finding, for a given matrix $\mathbf{A}$, all possible intensities $\Gamma$ for which system (26) is in equilibrium, we come to a fairly simple problem of the description of kernel of a linear operator assigned by the matrix A . Exactly this approach was used in [22] for finding symmetric equilibrium configurations. We give here a brief review of the found equilibrium configurations.

Tetrahedron $(N=4)$. In the case where the vortices under consideration are located at the vertices of a tetrahedron, we obtain that all $\ell_{i j}$ are equal between themselves, and, therefore, all elements of the matrix $\mathbf{A}$ are equal to zero. Such a degenerate matrix has a kernel of dimension nullity $(\mathbf{A})=N=4$, where nullity $(\mathbf{A})=\operatorname{dim}(\operatorname{ker}(\mathbf{A}))$, i.e., one can choose the basis of the kernel in the form $(1,0,0,0),(0,1,0,0)$, $(0,0,1,0),(0,0,0,1)$. This means that an arbitrary set of intensities $\left(\Gamma_{1}, \Gamma_{2}, \Gamma_{3}, \Gamma_{4}\right)$ will be contained in the kernel and will represent an equilibrium configuration. In other words, if, at the initial moment, vortices of an arbitrary intensity were located at the vertices of a tetrahedron, they will remain at these vertices. This result is demonstrated in Fig. 1. 

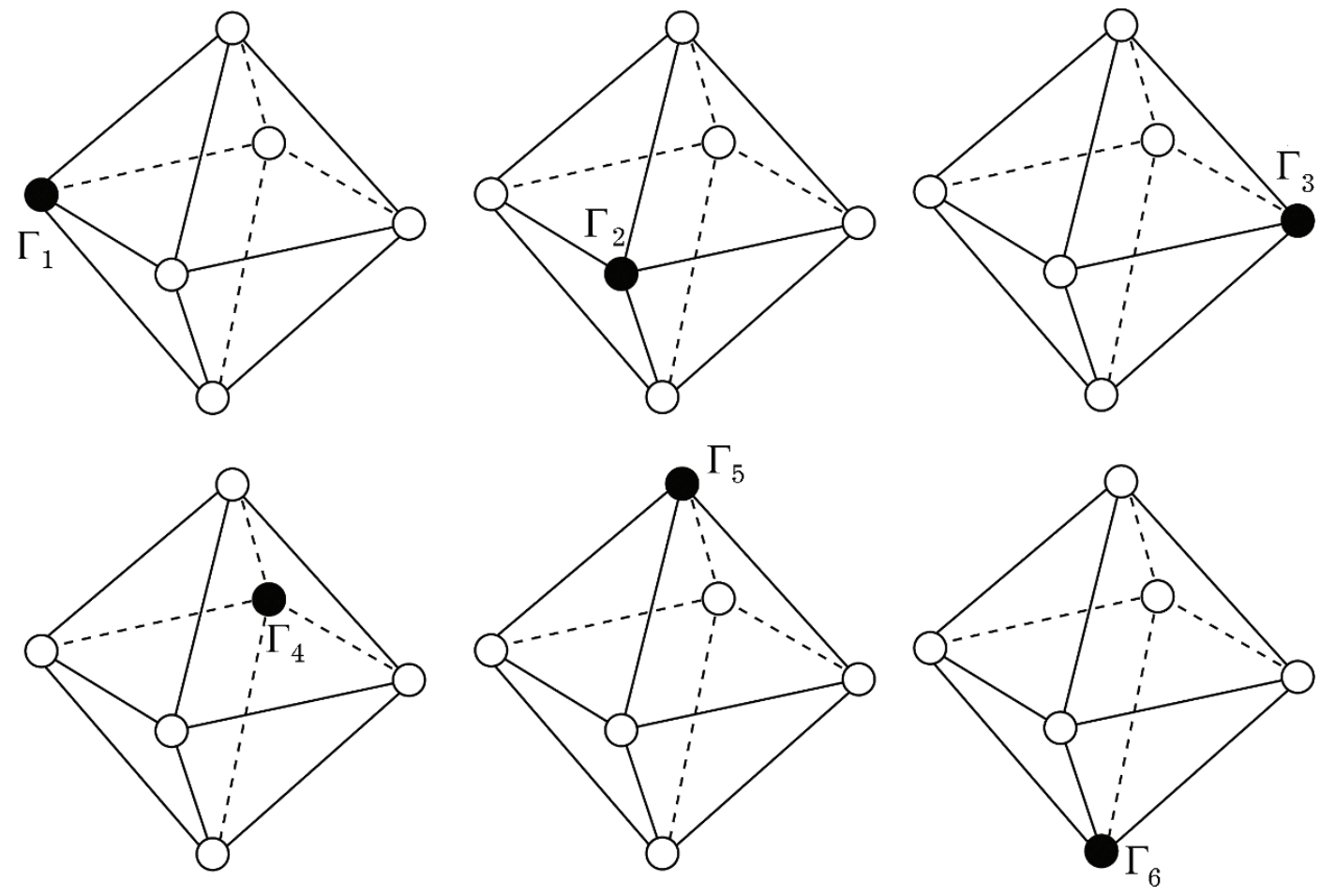

Fig. 2. Set of independent intensities generating an equilibrium configuration in the form of an octahedron.

Octahedron $(N=6)$. In the case where the vortices are located at the vertices of an octahedron, we establish that either the distances between each three vortices are identical, or they are situated in one plane. Therefore, all elements of the matrix $\mathbf{A}$ are also equal to zero. Considerations similar to those presented in the case of a tetrahedron enable us to conclude that, if vortices of an arbitrary intensity were located at the vertices of an octahedron at the initial moment, they will remain at these vertices (Fig. 2).

Cube $(N=8)$. In the case of a cube, the matrix $\mathbf{A}$ is already nondegenerate. We obtain that its kernel has dimension nullity $(\mathbf{A})=5$. The basis of kernel can be chosen in the form

$$
\begin{array}{r}
\operatorname{ker}(\mathbf{A})=\operatorname{span}((1,0,0,0,0,0,-1,0),(0,1,0,0,0,0,0,-1),(0,0,1,0,0,1,1,1), \\
(0,0,0,1,0,-1,0,0),(0,0,0,0,1,1,1,1)) .
\end{array}
$$

The numbering of vertices and the appearance of basis are shown in Figs. 3 and 4.

Antisymmetric cube $(N=8)$. In the case of an antisymmetric cube (a cube whose lower face is turned by $\pi / 4)$, the matrix $\mathbf{A}$ is also nondegenerate. Its kernel has a dimension nullity $(\mathbf{A})=1$. One can choose the basis of the kernel as $(1,1,1,1,-1,-1,-1,-1)$, where the first four coordinates describe the vortex intensities at the vertices of the upper face, and the last four describe the intensities at the vertices of the lower (turned) face. The graphic demonstration of this basis is presented in Fig. 5. As is seen in this figure, we have the classical configuration of von Kárman vortices. It should be noted that the von Kárman configurations will be equilibrium not only for an antisymmetric cube. 


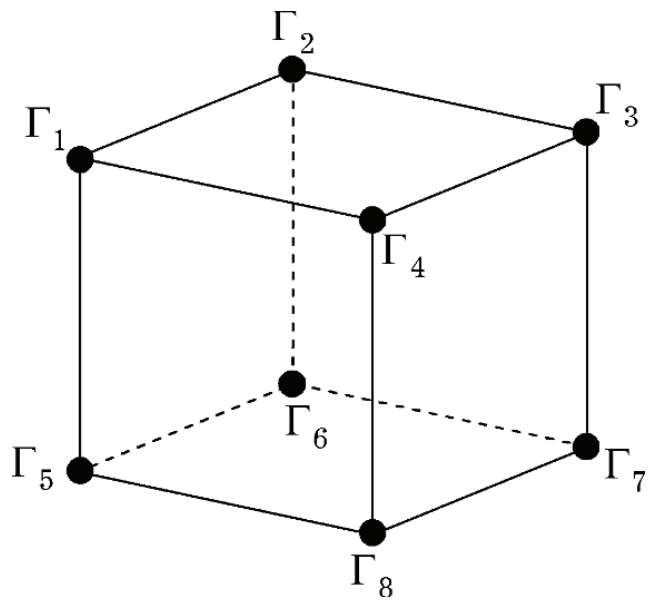

Fig. 3. Numbering of cube vertices.
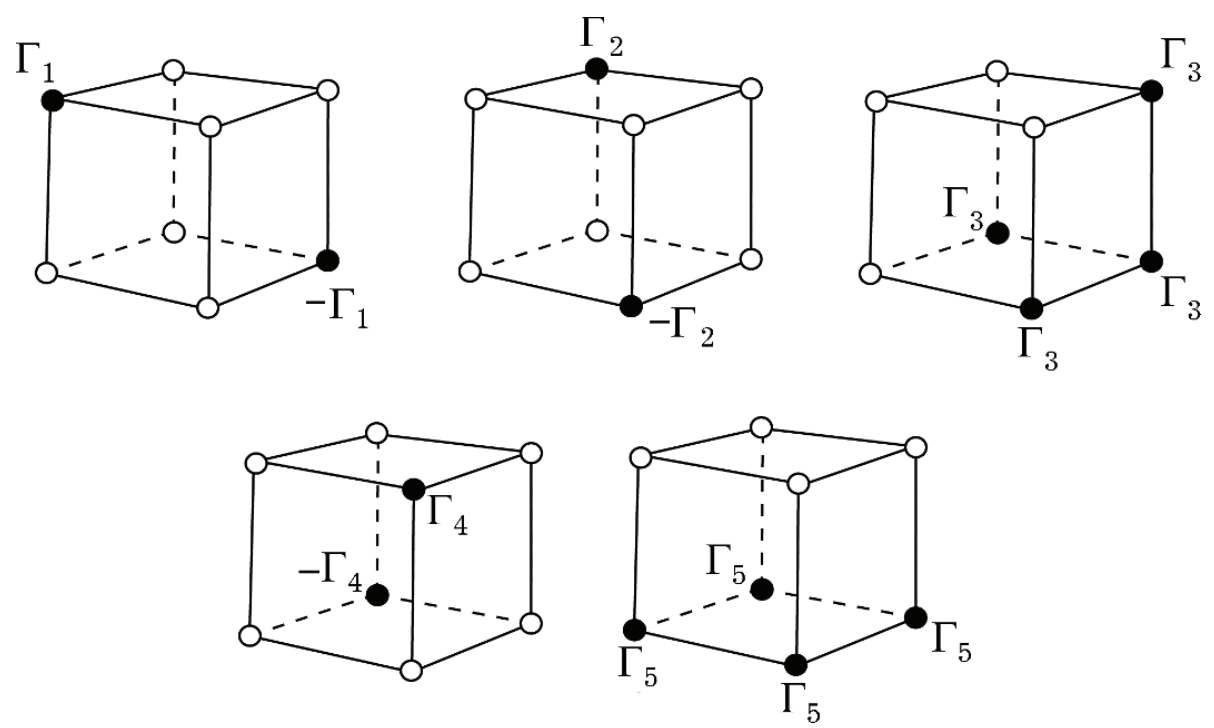

Fig. 4. Set of independent intensities generating an equilibrium configuration in the form of a cube.

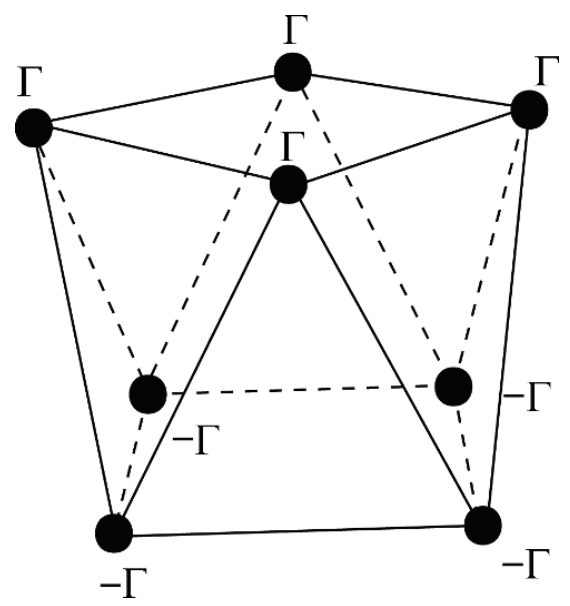

Fig. 5. A unique set of intensities generating an equilibrium configuration in the form of an antisymmetric cube. 


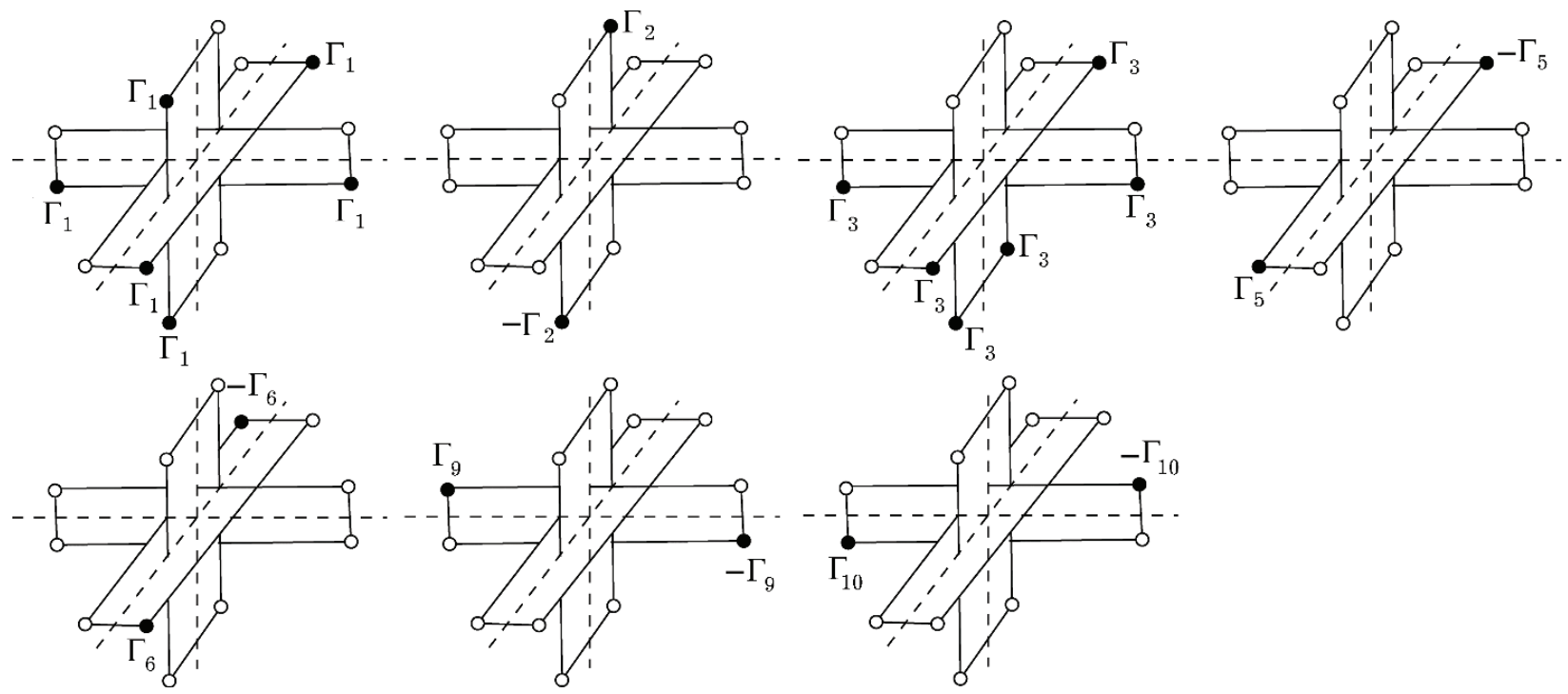

Fig. 6. Set of independent intensities generating an equilibrium configuration in the form of an icosahedron.

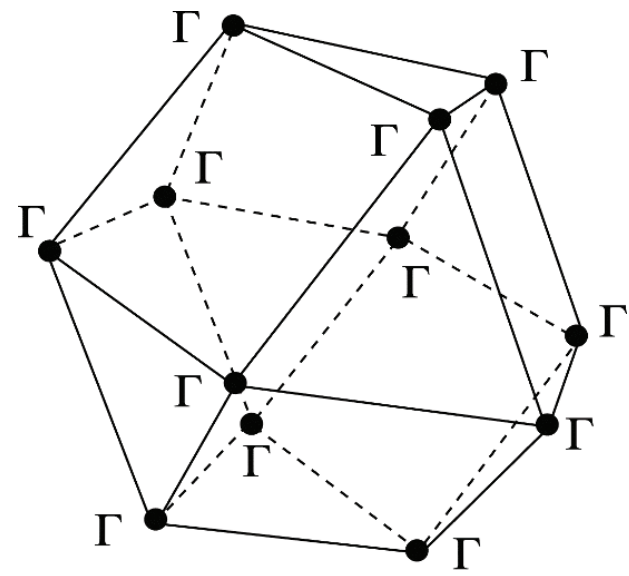

Fig. 7. Equilibrium configuration in the form of a cubic octahedron.

Icosahedron $(N=12)$. In the case of an icosahedron, we also have a nondegenerate matrix $\mathbf{A}$ with the dimension of its kernel nullity $(\mathbf{A})=7$. The explicit form of basis can be found in $[22,29]$. Its graphic representation is given in Fig. 6.

Dodecahedron $(N=20)$. If the vortices under study are located at the vertices of a dodecahedron, we obtain a nondegenerate matrix $\mathbf{A}$ with dimension of its kernel nullity $(\mathbf{A})=4$. The explicit form of the basis and its graphic representation are given in [22, 29].

Cubic Octahedron $(N=12)$. A cubic octahedron is an inscribed polyhedron depicted in Fig. 7. It is one of the so-called semiregular polyhedrons, or, as they are often called, Archimedian bodies. If the vortices are located at the vertices of a cubic octahedron, we obtain a nondegenerate matrix $\mathbf{A}$ with dimension of its kernel nullity $(\mathbf{A})=1$. The basis of kernel is described by a single vector, all elements of which are equal to unity, i.e., a cubic octahedron will be an equilibrium configuration only in the case where the intensities of all vortices are identical. 


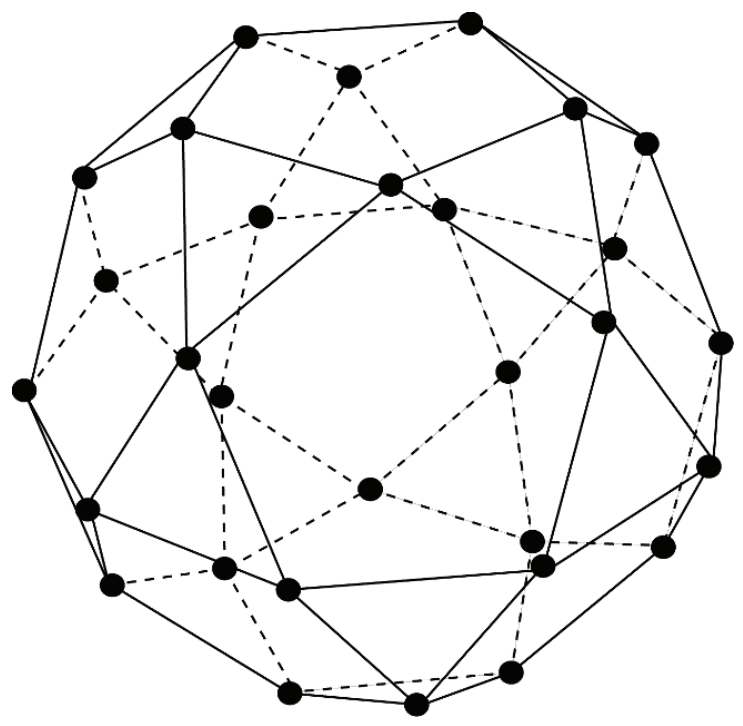

Fig. 8. Equilibrium configuration in the form of an icosidodecahedron.

Icosidodecahedron $(N=30)$. An icosidodecahedron represents one more semiregular convex inscribed polyhedron shown in Fig. 8. If the vortices are located at the vertices of an icosidodecahedron, we have a case similar to the previous one, i.e., a nondegenerate matrix $\mathbf{A}$ with dimension of its kernel nullity $(\mathbf{A})=1$. The basis of the kernel is described by a single vector, all elements of which are equal to unity, i.e., an icosidodecahedron will be an equilibrium configuration only in the case where the intensities of all vortices are identical.

Note that only two of the semiregular polyhedrons presented above are equilibrium configurations. In addition, we found several configurations in the form of superpositions of symmetric configurations that are also equilibrium.

Using the method described in this section, one can construct a numerical algorithm that makes it possible to find nonsymmetric equilibrium configurations [30].

\section{Stability of Equilibrium Configurations}

As noted in [11], there are numerous definitions of the stability of configurations of relative equilibrium. In the present work, we use the definition of stability according to Routh: a configuration of relative equilibrium is stable if, for an arbitrary $\varepsilon>0$, there exists $\delta>0$ such that any trajectory beginning in a $\delta$-neighborhood of the equilibrium configuration remains in an $\varepsilon$-neighborhood of this configuration.

For investigating the stability of configurations of relative equilibrium, we apply the criterion formulated in [11]: if the Hamiltonian in coordinates moving together with the system under consideration reaches its transversal minimum or maximum in the equilibrium configuration, then this configuration is stable. In this context, transversal minimum is the minimum of a function in the directions transversal to directions that keep the system invariable (the orbits of a symmetry group of the Hamiltonian).

To prove the instability of equilibrium configurations, we use the well-known fact from the theory of stability of linear systems: if, among the eigenvalues of matrix linearized near the equilibrium configuration of the system, there are numbers with positive real part, then the original system is unstable.

To simplify our calculations, we use the canonical variables

$$
\varphi_{i}=\varphi_{i}, \quad z_{i}=\cos \theta_{i}
$$


The Hamiltonian of the system in these variables has the form

$$
H=\frac{1}{2} \sum_{i, j=1, i \neq j}^{N} \Gamma_{i} \Gamma_{j} \ln \left[1-z_{i} z_{j}-\sqrt{1-z_{i}^{2}} \sqrt{1-z_{j}^{2}} \cos \left(\varphi_{i}-\varphi_{j}\right)\right] .
$$

We take

$$
R_{i j}=\left(1-z_{i} z_{j}-\cos \left(\varphi_{i}-\varphi_{j}\right) \sqrt{1-z_{i}^{2}} \sqrt{1-z_{j}^{2}}\right)
$$

and, hence, the components of the matrix of the second derivatives are

$$
\begin{aligned}
& \frac{\partial^{2} H}{\partial \varphi_{i}^{2}}=\sum_{j \neq i j=1}^{N} \frac{\Gamma_{i} \Gamma_{j}}{2}\left(\frac{\cos \left(\varphi_{i}-\varphi_{j}\right) \sqrt{1-z_{i}^{2}} \sqrt{1-z_{j}^{2}}\left(1-z_{i} z_{j}\right)-\left(1-z_{i}^{2}\right)\left(1-z_{j}^{2}\right)}{R_{i j}^{2}}\right), \\
& \frac{\partial^{2} H}{\partial \varphi_{i} \partial \varphi_{j}}=\frac{\Gamma_{i} \Gamma_{j}}{2}\left(\frac{\cos \left(\varphi_{i}-\varphi_{j}\right) \sqrt{1-z_{i}^{2}} \sqrt{1-z_{j}^{2}}\left(z_{i} z_{j}-1\right)+\left(1-z_{i}^{2}\right)\left(1-z_{j}^{2}\right)}{R_{i j}^{2}}\right), \\
& \frac{\partial^{2} H}{\partial z_{i}^{2}}=\sum_{\substack{j=1 \\
j \neq i}}^{N} \frac{\Gamma_{i} \Gamma_{j}}{2}\left(\frac{\left(z_{j}\left(1-z_{i}^{2}\right)-\cos \left(\varphi_{i}-\varphi_{j}\right) z_{i} \sqrt{1-z_{j}^{2}}\right)^{2}-\cos \left(\varphi_{i}-\varphi_{j}\right) \sqrt{1-z_{j}^{2}} R_{i j}}{\left(1-z_{i}^{2}\right) \sqrt{1-z_{j}^{2}} R_{i j}^{2}}\right), \\
& \frac{\partial^{2} H}{\partial z_{i} \partial z_{j}}=\frac{\Gamma_{i} \Gamma_{j}}{2}\left(\frac{\cos \left(\varphi_{i}-\varphi_{j}\right) \sqrt{1-z_{i}^{2}} \sqrt{1-z_{j}^{2}}\left(z_{i} z_{j}-1\right)+\sqrt{1-z_{i}^{2}} \sqrt{1-z_{j}^{2}}}{\sqrt{1-z_{i}^{2}} \sqrt{1-z_{j}^{2}} R_{i j}^{2}}\right), \\
& \frac{\partial^{2} H}{\partial z_{i} \partial \varphi_{i}}=\sum_{j=1, j \neq i}^{N} \frac{\Gamma_{i} \Gamma_{j}}{2}\left(\frac{\sin \left(\varphi_{i}-\varphi_{j}\right) \sqrt{1-z_{i}^{2}}\left(z_{i}-z_{j}\right)}{\sqrt{1-z_{j}^{2}} R_{i j}^{2}}\right), \\
& \frac{\partial^{2} H}{\partial z_{j} \partial \varphi_{i}}=\frac{\Gamma_{i} \Gamma_{j}}{2}\left(\frac{\sin \left(\varphi_{i}-\varphi_{j}\right) \sqrt{1-z_{i}^{2}}\left(z_{i}-z_{j}\right)}{\sqrt{1-z_{j}^{2}} R_{i j}^{2}}\right) .
\end{aligned}
$$

The components of the linearized system are connected with the components of matrix of the second derivatives by the following relation:

$$
\frac{d}{d t}\left(\begin{array}{l}
\mathbf{z} \\
\boldsymbol{\varphi}
\end{array}\right)=\mathbf{L}_{2 N}\left(\begin{array}{l}
\mathbf{z} \\
\boldsymbol{\varphi}
\end{array}\right)=\left(\begin{array}{ll}
\mathbf{0}_{N} & \mathbf{I}_{N} \\
-\mathbf{I}_{N} & \mathbf{0}_{N}
\end{array}\right) \mathbf{A}_{2 N}\left(\begin{array}{l}
\mathbf{z} \\
\boldsymbol{\varphi}
\end{array}\right)
$$


Table 1. Coordinates of the Vertices and Eigenvalues of the Matrix of the Second Derivatives for Stable Configurations

\begin{tabular}{|c|c|}
\hline Coordinates of the vertices $\left(z_{i}^{0}, \varphi_{i}^{0}\right)$ & Eigenvalues of the matrix $\mathbf{A}_{2 N}$ \\
\hline \multicolumn{2}{|c|}{ Tetrahedron $(N=4)$} \\
\hline$\left((-1)^{k} \frac{1}{\sqrt{3}}, \frac{\pi k}{2}\right), \quad k=0, \ldots, 3$ & $-\frac{9}{4},-\frac{21}{16},-\frac{21}{16},-\frac{9}{8},-\frac{1}{2}, 0,0,0$ \\
\hline \multicolumn{2}{|c|}{ Octahedron $(N=6)$} \\
\hline $\begin{array}{l}\left(\frac{1}{\sqrt{2}}, 0\right),\left(\frac{1}{\sqrt{2}}, \pi\right),\left(-\frac{1}{\sqrt{2}}, 0\right) \\
\left(-\frac{1}{\sqrt{2}}, \pi\right),\left(0, \frac{\pi}{2}\right),\left(0, \frac{3 \pi}{2}\right)\end{array}$ & $\begin{array}{c}-4,-\frac{9+\sqrt{41}}{4},-\frac{9+\sqrt{41}}{4},-\frac{3}{2},-\frac{3}{2},-\frac{5}{4} \\
-\frac{9-\sqrt{41}}{4},-\frac{9-\sqrt{41}}{4},-\frac{1}{4}, 0,0,0\end{array}$ \\
\hline & $\begin{array}{l}\text { Numerical approximations of the eigenvalues of the } \\
\text { matrix } \mathbf{A}_{2 N}\end{array}$ \\
\hline \multicolumn{2}{|c|}{ Icosahedron $(N=12)$} \\
\hline $\begin{array}{c}\left((-1)^{k} \sqrt{\frac{5+\sqrt{5}}{10}},(-1)^{\ell} \frac{\pi}{2}\right), \\
\left((-1)^{k} \sqrt{\frac{5-\sqrt{5}}{10}}, \pi^{\ell}\right), \\
\left(0,(-1)^{k}\left(\pi^{\ell}-\arccos \sqrt{\frac{5-\sqrt{5}}{10}}\right)\right), \\
k=0,1, \ell=0,1\end{array}$ & $\begin{array}{c}-23.8462,-22.9415,-17.8963 \\
-12.0918,-9.73771,-9.69726 \\
-9.03378,-5.9006,-5.59474 \\
-5.0765,-4.70151,-3.51885 \\
-2.8623,-2.44798,-2.22719 \\
-1.22744,-1.19444,-0.877398 \\
-0.680072,-0.551888,-0.281533 \\
0,0,0\end{array}$ \\
\hline
\end{tabular}

where $\mathbf{z}$ and $\boldsymbol{\varphi}$ are the column vectors of coordinates $z_{i}, \varphi_{i}, i=1, \ldots, N, \mathbf{0}_{N}$, and $\mathbf{I}_{N}$ are the zero and unit $N \times N$-matrices, and $\mathbf{A}_{2 N}=D^{2} H\left(z_{0}, \varphi_{0}\right)$ is the matrix of the second derivatives in the equilibrium configuration.

Since the equilibrium configurations are critical points of the Hamiltonian, it follows from the transversal positive (negative) definiteness of the second variation that the Hamiltonian reaches its transversal minimum (maximum) in the equilibrium configuration. In turn, to prove that a matrix is transversally positive (negative) defined, it is sufficient to show that the eigenvalues corresponding to directions transversal to the equilibrium configurations are positive (negative). Note that the eigenvalues corresponding to directions tangential to the orbits of the equilibrium configurations have to be zero.

To simplify our calculations in the case of equal intensities, we may take without loss of generality $\Gamma_{i}=1$, $i=1, \ldots, N$.

Using these considerations, we prove the following theorems:

Theorem 2. In the case of equal intensities, the equilibrium configurations in the form of a tetrahedron, an octahedron, and an icosahedron are nonlinearly stable. 
Proof. Since the configurations of relative equilibrium mentioned above are fixed degenerate equilibrium configurations ( $\mathbf{M}=\mathbf{0}$ ), we use, for studying stability, the initial fixed coordinate system, where the Hamiltonian has the form (32). The coordinates of vertices and eigenvalues of the matrix $\mathbf{A}_{2 N}$ for these cases are given in Table 1.

The symmetry group that does not change these equilibrium configurations is the group $\mathrm{SO}(3)$ (arbitrary rotation of a sphere does not disturb equilibrium configurations). The space tangential to the orbit of this group is three-dimensional, which explains the presence of three zero eigenvalues. All eigenvalues corresponding to the transversal directions are negative, and, hence, according to the criterion presented above, the equilibrium configurations under study are stable.

Theorem 3. In the case of equal intensities, equilibrium configurations in the form of a cube, a dodecahedron, a cubic octahedron, and an icosidodecahedron are unstable.

Proof. Since these configurations of relative equilibrium are also fixed degenerate equilibrium configurations $(\mathbf{M}=\mathbf{0})$, the Hamiltonian, as follows from the considerations presented above, has the form (32). The coordinates of vertices and eigenvalues of the matrix $\mathbf{L}_{2 N}$ are given in Table 2. Among the eigenvalues of each configuration, there are those whose real part is positive, and, hence, these configurations are unstable.

Theorem 4. Equilibrium configuration in the form of an antisymmetric cube is unstable.

Proof. In the equilibrium configuration under study, not all intensities are equal between themselves. Without loss of generality, we take $\Gamma_{i}=1, \Gamma_{j}=-1, i=1, \ldots, 4, j=5, \ldots, 8$. Such a configuration of relative equilibrium is nondegenerate $(\mathbf{M} \neq \mathbf{0})$, and all vortices will rotate around the vector $\mathbf{M}$. We choose a coordinate system where the vector $\mathbf{M}$ is codirectional with the positive direction of $O z$ axis. The coordinates of vertices of an antisymmetric cube in the chosen coordinate system are presented in Table 2.

As noted above, for studying the stability of the system, it is necessary to pass to a coordinate system rotating with the vortices. The Hamiltonian in such a moving coordinate system has the form

$$
H_{1}=H+\omega \sum_{i=1}^{N} z_{i},
$$

where $H$ is Hamiltonian (32) and $\omega$ is the angular velocity of rotation of the configuration. Since the equilibrium configuration is a critical point of the Hamiltonian $H_{1}$, we can find the angular velocity $\omega$ as the solution of the equation

$$
\frac{\partial H_{1}}{\partial z_{i}}\left(\left(z_{i}^{0}, \varphi_{i}^{0}\right)=-\frac{41 \sqrt{3}}{28}+\omega\right)=0
$$

i.e.,

$$
\omega=\frac{41 \sqrt{3}}{28}
$$

Since the Hamiltonian $H_{1}$ depends linearly on the coordinates $z_{i}$, we have

$$
D^{2} H_{1}=D^{2} H
$$


Table 2. Coordinates of the Vertices and Eigenvalues of the Linearized System for Unstable Configurations

\begin{tabular}{|c|c|}
\hline Coordinates of the vertices $\left(z_{i}^{0}, \varphi_{i}^{0}\right)$ & Eigenvalues of the matrix $\mathbf{L}_{2 N}$ \\
\hline \multicolumn{2}{|r|}{ Cube $(N=8)$} \\
\hline $\begin{aligned}\left(\frac{1}{\sqrt{3}}, \frac{\pi k}{2}\right) & ,\left(-\frac{1}{\sqrt{3}}, \frac{\pi k}{2}\right) \\
k & =0, \ldots, 3\end{aligned}$ & $\begin{array}{c}i \sqrt{3}, i \sqrt{3},-i \sqrt{3},-i \sqrt{3}, \frac{\sqrt{15}}{4}, \frac{\sqrt{15}}{4} \\
-\frac{\sqrt{15}}{4},-\frac{\sqrt{15}}{4}, 0,0,0,0\end{array}$ \\
\hline & Numerical approximations of the eigenvalues of the matrix $\mathbf{L}_{2 N}$ \\
\hline \multicolumn{2}{|r|}{ Antisymmetric cube $(N=8)$} \\
\hline $\begin{array}{c}\left(\frac{1}{\sqrt{3}}, \frac{\pi k}{2}\right),\left(-\frac{1}{\sqrt{3}}, \frac{\pi+2 \pi k}{4}\right) \\
k=0, \ldots, 3\end{array}$ & $\begin{array}{c}-1.50425,-1.50425,1.50425,1.50425,1.41833 \\
-1.41833,1.41833,-1.41833,0.986012 i \\
-0.986012 i, 0.986012 i,-0.986012 i, 0.809152 \\
-0.809152,0,0\end{array}$ \\
\hline \multicolumn{2}{|r|}{ Dodecahedron $(N=20)$} \\
\hline $\begin{array}{c}\left(\alpha, \frac{\pi+2 \pi k}{5}\right),\left(\pi-\alpha, \frac{2 \pi k}{5}\right) \\
\left(\beta, \frac{\pi}{5}+\frac{2 \pi k}{5}\right),\left(\pi-\beta, \frac{2 \pi k}{5}\right) \\
\alpha=\arccos \sqrt{\frac{5+2 \sqrt{5}}{15}} \\
\beta=\alpha+2 \arccos \sqrt{\frac{3+\sqrt{5}}{6}} \\
k=0, \ldots, 4\end{array}$ & $\begin{array}{c}4.66871 i,-4.66871 i, 4.66871 i,-4.66871 i \\
4.66871 i,-4.66871 i, 4.66871 i,-4.66871 i \\
4.6614 i,-4.6614 i, 4.6614 i,-4.6614 i, 4.6614 i \\
-4.6614 i, 4.6614 i,-4.6614 i, 4.6614 i,-4.6614 i \\
3.12812,-3.12812,3.12812,-3.12812,3.12812 \\
-3.12812,2.72398,-2.72398,2.72398,-2.72398 \\
2.72398,-2.72398,2.72398,-2.72398,2.72398 \\
-2.72398,0,0,0,0,0,0\end{array}$ \\
\hline \multicolumn{2}{|r|}{ Cubic octahedron $(N=12)$} \\
\hline $\begin{array}{c}\left(\frac{1}{\sqrt{2}}, \frac{\pi k}{2}\right),\left(-\frac{1}{\sqrt{2}}, \frac{\pi k}{2}\right), \\
\left(0, \frac{\pi+2 \pi k}{4}\right),\end{array}$ & $\begin{array}{c}\frac{2 \sqrt{134}}{9} i,-\frac{2 \sqrt{134}}{9} i, \frac{2 \sqrt{134}}{9} i,-\frac{2 \sqrt{134}}{9} i, \frac{2 \sqrt{134}}{9} i \\
-\frac{2 \sqrt{134}}{9} i, \frac{\sqrt{35}}{3} i,-\frac{\sqrt{35}}{3} i, \frac{\sqrt{35}}{3} i,-\frac{\sqrt{35}}{3} i, \frac{2 \sqrt{2}}{3} i \\
-\frac{2 \sqrt{2}}{3} i, \frac{2 \sqrt{2}}{3} i,-\frac{2 \sqrt{2}}{3} i, \frac{2 \sqrt{2}}{3} i,-\frac{2 \sqrt{2}}{3} i, \frac{\sqrt{37}}{3} \\
-\frac{\sqrt{37}}{3}, 0,0,0,0,0,0\end{array}$ \\
\hline
\end{tabular}


Table 2

(continued)

\begin{tabular}{|c|c|}
\hline Coordinates of the vertices $\left(z_{i}^{0}, \varphi_{i}^{0}\right)$ & Eigenvalues of the matrix $\mathbf{L}_{2 N}$ \\
\hline \multicolumn{2}{|c|}{ Icosidodecahedron $(N=30)$} \\
\hline $\begin{array}{c}\left(0, \frac{\pi+2 \pi k}{10}\right), \\
\left(-\sqrt{\frac{5+\sqrt{5}}{10}}, \frac{2 \pi \ell}{5}\right), \\
\left(\sqrt{\frac{5+\sqrt{5}}{10}}, \frac{\pi+2 \pi \ell}{5}\right), \\
\left(\sqrt{\frac{5-\sqrt{5}}{10}}, \frac{2 \pi \ell}{5}\right) \\
\left(-\sqrt{\frac{5-\sqrt{5}}{10}}, \frac{\pi+2 \pi \ell}{5}\right), \\
k=0, \ldots, 9, \ell=0, \ldots, 4\end{array}$ & $\begin{array}{c}7.15642 i,-7.15642 i, 7.15642 i,-7.15642 i, \\
7.15642 i,-7.15642 i, 6.80416,-6.80416,6.80416, \\
-6.80416,6.80416,-6.80416,6.57547 i, \\
-6.57547 i, 6.57547 i,-6.57547 i, 6.57547 i, \\
-6.57547 i, 6.57547 i,-6.57547 i, 6.57547 i, \\
-6.57547 i, 5.98329,-5.98329,5.98329,-5.98329, \\
-5.98329,5.98329,-5.98329,5.98329,5.87649 i, \\
-5.87649 i, 5.87649 i,-5.87649 i, 5.87649 i, \\
-5.87649 i, 2.76964 i,-2.76964 i, 2.76964 i, \\
-2.76964 i, 2.76964 i,-2.76964 i, 2.76964 i \\
-2.76964 i, 2.38582 i,-2.38582 i, 2.38582 i, \\
-2.38582 i, 2.38582 i,-2.38582 i, 2.38582 i, \\
-2.38582 i, 2.38582 i,-2.38582 i, 0,0,0,0,0,0\end{array}$ \\
\hline
\end{tabular}

i.e., the second variation of the Hamiltonian will not change in going to the moving coordinate system. The eigenvalues of matrix $\mathbf{L}_{2 N}$ are given in Table 2. Among the eigenvalues of this configuration, there are those whose real part is positive, and, hence, the configuration under study is unstable.

It is worth noting that the described results concerning the stability of equilibrium configurations in the form of a tetrahedron, an octahedron, and an icosahedron as well as the instability of a cube and a dodecahedron agree completely with the data [11]. The instability of equilibrium configurations in the form of an antisymmetric cube and a cube are special cases of the analysis of stability of circular pairs considered in [26].

\section{Concluding Remarks}

We see that the methods of linear algebra enable one to verify quite simply whether a given configuration of point vortices on a sphere is a configuration of relative equilibrium. These methods can be generalized for the case of search for plane configurations of relative equilibrium.

For studying stability, it is necessary to focus on the general theory of stability of Hamiltonian systems with symmetries. Although the problem of finding the symmetries of a system quite often is the most complex part of investigation, in the case of point vortices on a sphere, its rotation symmetries are natural symmetries. If the symmetries are known, the problem of studying stability is reduced to investigation of the second variation of the Hamiltonian in equilibrium configuration and of linearized system near this configuration.

\section{REFERENCES}

1. S. V. Alekseenko, P. A. Kuibin, and V. L. Okulov, An Introduction to the Theory of Concentrated Vortices [in Russian], Institute of Thermophysics, Siberian Branch, Russ. Acad. Sci., Novosibirsk (2003). 
2. V. A. Bogomolov, "Vorticity dynamics on a sphere," Izv. Akad. Nauk SSSR, Mekh. Zhidk. Gaza, No. 6, 57-65 (1977).

3. A. V. Borisov and I. S. Mamaev, Mathematical Methods of the Dynamics of Vortex Structures [in Russian], Inst. Comput. Res., Moscow (2005).

4. A. V. Borisov, L. A. Gazizullina, and S. M. Ramodanov, "The thesis by E. Zermelo on the vortex hydrodynamics on a sphere," Nelin. Dyn., 4, 497-513 (2008).

5. O. F. Vasil'ev, “On one forgotten work by I. S. Gromeka,” Prikl. Mat. Mekh., 15, 261-263 (1951).

6. H. Villat, Leçons sur la Théorie des Tourbillons, Gauthier-Villars, Paris (1930).

7. I. S. Gromeka, On the Vortex Motions of a Fluid on a Sphere [in Russian], Kazan' Univ., Kazan' (1885).

8. A. Sommerfeld, Mechanics of Deformable Bodies, Academic Press, New York (1950).

9. G. Kirchhoff, Vorlesungen über Mathematische Physik, Bd. 1: Mechanik, Leipzig (1876).

10. V. V. Kozlov, General Theory of Vortices [in Russian], Udmurt. Univ., Izhevsk (1998).

11. L. G. Kurakin, "On the nonlinear stability of regular vortex polygons and polyhedrons on a sphere," Dokl. Ross. Akad. Nauk, 388, 482-487 (2003).

12. V. V. Meleshko and M. Yu. Konstantinov, Dynamics of Vortex Structures [in Russian], Naukova Dumka, Kiev (1993).

13. V. V. Ostrovs'kyi and V. V. Meleshko, "Dynamics of point vortices on a sphere," Visn. Kyiv. Univ., Ser. Fiz.-Mat. Nauky, No. 3, 23-28 (2007).

14. V. V. Ostrovs'kyi, "Fluid motion in the field of a vortex pair on a fixed sphere," Visn. Kyiv. Univ., Ser. Fiz.-Mat. Nauky, No.4, 2226 (2008).

15. H. Poincaré, Theory of Vortices [Russian translation], RKhD, Izhevsk (2001).

16. P. G. Saffman, Vortex Dynamics, Cambridge University Press, Cambridge (1992).

17. H. Aref, P. K. Newton, M. A. Stremler, et al., "Vortex crystals," Adv. Appl. Mech., 39, 1-79 (2003).

18. A. V. Borisov and V. G. Lebedev, "Dynamics of three vortices on a plane and a sphere. II. General compact case," Regular Chaotic Dyn., 3, No. 2, 99-114 (1998).

19. A. V. Borisov and V. G. Lebedev, "Dynamics of three vortices on a plane and a sphere. III. Noncompact Case. Problems of Collaps and Scattering," Regular Chaotic Dyn., 3, No.4, 74-86 (1998).

20. H. Cabral, K. Meyer, and D. Schmidt, "Stability and bifurcations for the $N+1$ vortex problem on the sphere," Regular Chaotic Dyn., 8, 259-282 (2003).

21. H. Helmholtz, "Über Integrale der hydrodynamischen Gleichungen, welche den Wirbelbewegungen entsprechen," J. Reine Angew. Math., 55, 25-55 (1858).

22. M. I. Jamaloodeen and P. K. Newton, "The $N$-vortex problem on a rotating sphere. II. Heterogeneous Platonic solid equilibria," Proc. Roy. Soc. London, A462, 3277-3299 (2006).

23. Y. Kimura, "Vortex motion on surfaces with constant curvature," Proc. Roy. Soc. London, A455, 245-259 (1999).

24. Y. Kimura and H. Okamoto, "Vortex motion on a sphere," J. Phys. Soc. Jpn., 56, 4203-4206 (1987).

25. L. G. Kurakin and V. I. Yudovich, "The stability of stationary rotation of a regular vortex polygon," Chaos, 12, 574-595 (2002).

26. F. Laurent-Polz, "Point vortices on the sphere: a case with opposite vorticities," Nonlinearity, 15, 143-171 (2002).

27. D. Lewis and T. Ratiu, "Rotating $n$-gon/ kn -gon vortex configurations," J. Nonlin. Sci., 6, 385-414 (1996).

28. V. V. Meleshko and H. Aref, “A bibliography of vortex dynamics 1858-1956," Adv. Appl. Mech., 41, 197-292 (2007).

29. P. K. Newton, The N-Vortex Problem. Analytical Techniques, Springer, New York (2001).

30. P. K. Newton and G. Chamoun, "Construction of point vortex equilibria via Brownian ratchets," Proc. Roy. Soc. London, A463, 1525-1540 (2007).

31. P. K. Newton and H. Shokraneh, "The $N$-vortex problem on a rotating sphere. I. Multifrequency configurations," Proc. Roy. Soc. London, A462, 149-169 (2006).

32. S. Pekarsky and J. E. Marsden, "Point vortices on a sphere: stability of relative equilibria," J. Math. Phys., 39, 5894-5907 (1998).

33. J. L. Synge, "On the motion of three vortices," Can.J. Math., 1, 257-270 (1949).

34. E. Zermelo, "Hydrodynamische Untersuchungen über die Wirbelbewegung in einer Kügelfläche,” Z. Math. Phys., 47, 201-237 (1902). 